\title{
Optimum sizing of bare-tape tethers for de-orbiting satellites at end of mission
}

\author{
J.R. Sanmartín*, A .Sánchez-Torres, S.B. Khan, G. Sánchez-Arriaga, M. Charro
}

\begin{abstract}
De-orbiting satellites at end of mission would prevent generation of new space debris. A proposed de-orbit technology involves a bare conductive tape-tether, which uses neither propellant nor power supply while generating power for on-board use during de-orbiting. The present work shows how to select tape dimensions for a generic mission so as to satisfy requirements of very small tether-to-satellite mass ratio $m_{t} / M_{S}$ and probability $N_{f}$ of tether cut by small debris, while keeping de-orbit time $t_{f}$ short and product $t_{f} \times$ tether length low to reduce maneuvers in avoiding collisions with large debris. Design is here discussed for particular missions (initial orbit of $720 \mathrm{~km}$ altitude and $63^{\circ}$ and $92^{\circ}$ inclinations, and 3 disparate $M_{S}$ values, $37.5,375$, and $3750 \mathrm{~kg}$ ), proving it scalable. At mid-inclination and a mass-ratio of a few percent, de-orbit time takes about 2 weeks and $N_{f}$ is a small fraction of $1 \%$, with tape dimensions ranging from 1 to $6 \mathrm{~cm}$, 10 to $54 \mu \mathrm{m}$, and 2.8 to $8.6 \mathrm{~km}$. Performance drop from middle to high inclination proved moderate: if allowing for twice as large $m_{t} / M_{S}$, increases are reduced to a factor of 4 in $t_{f}$ and a slight one in $N_{f}$, except for multi-ton satellites, somewhat more requiring because efficient orbital-motion-limited electron collection restricts tape-width values, resulting in tape length (slightly) increasing too.
\end{abstract}

\section{Introduction}

Space debris remains a constant menace to operative satellites in Low Earth Orbit (LEO), the risk in setting up the well-known Kessler cascade increasing with time (Kessler and Cour-Palais, 1978). Future satellites should thus incorporate a de-orbit system to be used just at end of mission. Electrodynamic tethers, which are propellantless and passive systems using Lorentz drag by geomagnetic field $\mathbf{B}$ on tether current driven by the motional field $\mathbf{E}_{\mathbf{m}}$ induced by $\mathbf{B}$ itself, might effectively remove both future and current non-active satellites (Forward et al., 1998; Van der Heide and Kruijff, 2001; Ahedo and Sanmartin, 2002;
Gilchrist et al., 2002; Pardini et al., 2009, 2006). Any de-orbiting system faces two basic requirements: it must (i) be light when compared to its satellite, and (ii) operate fast to avoid its accidental, catastrophic collision with another large orbiting object, resulting in a myriad of debris pieces. A tether system also faces three particular issues: (a) it might be somewhat ineffective at high inclination orbits for which $\mathbf{E}_{\mathbf{m}}$ could prove too weak; $(b)$ its geometry (long and thin) make it prone to being cut by abundant tiny debris, leading to a failed operation; and $(c)$ its geometry (long) might make it also prone to cut by a big debris.

As regards point $(b)$ above, recent results showed that tape tethers have much greater survival probability than round tethers of equal length and mass (Khan and Sanmartin, 2013). Tether geometry has thus a relevant impact on system performance, and tape tethers are advantageous in this respect. Given a mission, i. e. the initial 
orbital parameters and the mass of the satellite, one might choose tether length $L$, width $w$, and thickness $h$ to optimize some figure of merit. Opposite requirements of both a light tether and survivability against debris suggest a design scheme based on the product $\Pi$ of probability $N_{f}$ of a cut and tether-to-satellite mass ratio $m_{t} / M_{S}$. Optimal tether design will hinge on both a minimum of the dimensionless function $I I$ and short de-orbiting to reduce manouvers in avoiding big tracked debris.

The present work explicitly shows $\Pi$ as a functional of tether geometry and orbital parameters, which is derived by combining a fatal-impact rate model introduced in Khan and Sanmartin (2014) and a simple satellite dynamical equation, which assumes a slow de-orbit evolution as sequence of near-circular orbits. Product $\Pi$, involving Lorentz drag and space debris impacts, just depends on mission constraints and tether dimensions.

Results from the algorithm highlight important features of bare-tether technology. It is scalable, allowing it to be competitive for a satellite mass range from tens of kilograms to multiple tons, and high inclination effects are moderate. This is illustrated by applying the design algorithm to hypothetical missions for de-orbiting satellites from the Cryosat orbit. Cryosat, an operative Earth-observing satellite following a non-synchronous orbit at $720 \mathrm{~km}$ altitude and $92^{\circ}$ inclination, was launched in April 2010 to measure polar ice thickness. The algorithm is applied to $37.5,375$, and $3750 \mathrm{~kg}$ satellites, and it is also similarly applied for $63^{\circ}$ inclination.

In Sections 2 and 3 of the paper the tether survival probability model obtained in Khan and Sanmartin (2014) and the simple deorbit dynamical equation are presented, respectively. These results are combined in Section 4 to obtain both function $\Pi$ and de-orbit time $t_{f}$. Applications of the optimization algorithm are discussed in Section 5 and conclusions are summarized in Section 6.

\section{Survival against debris}

As already mentioned, results found by Khan and Sanmartin (2013) show that thin-tape conductive tethers have much greater survival probability than round tethers of equal length $L$ and mass $m_{c t}$ of conductive segment. High survival probability over a de-orbit time $\Delta t$ requires a low fatal-impact final count $N_{f}$ in a Poisson probability distribution

$P \approx \exp \left(-N_{f}\right) \approx 1-N_{f}$,

where $N_{f}$, in case of constant conditions, would be simply related to the fatal-impact count rate, $N_{f} / L \Delta t=\dot{n}_{c}$. A value $N_{f}=0.05$, say, would mean estimating that 5 among 100 tethers would be cut while de-orbiting.

For the simplest case of a round tether of diameter $D$, a standard approximation for the fatal impact rate reads

$\frac{d N_{c}}{d t}=-\int_{\delta_{m(D)}}^{\delta_{\infty}} \frac{d F}{d \delta} d \delta \times L D_{e f f}(D, \delta)$, where $F(\delta)$ is the cumulative flux down to debris size $\delta$, at given orbit altitude and inclination, by either ESA's MASTER (Flegel et al., 2009) or NASA's ORDEM (Liou et al., 2002) flux models. In Eq. (2), $\delta_{\infty}$ is a largest debris size relevant as regards cuts, say $1 \mathrm{~m}, \delta_{m}(D)$ is the minimum size that may sever a tether, and $D_{\text {eff }}=D+\delta-\delta_{c}$ is an effective tether diameter for debris collision, which takes into account that debris have macroscopic size and that severing requires a minimum volume overlap of tether and debris trajectories. Energy considerations suggest representative values $\delta_{c}=\delta_{m}=D / 3$.

For tapes, the fatal impact rate involves an additional integral over impact angle between debris velocity and normal to the wide side of the tape. For a tape-tether of length $L$, width $w$, and thickness $h$, Khan \& Sanmartin, making simple approximations, found an analytical representation for either MASTER or ORDEM models

$$
\begin{aligned}
d N_{c} / d t \equiv L \dot{n}_{c} \approx L \delta^{*} F^{*} \times G\left(n_{0}, n_{1}, \delta^{*} / w, w / h\right), & \\
G \equiv & \frac{3 n_{0}+2}{\pi\left(n_{0}-2\right)}\left(\frac{3 \delta^{*}}{w}\right)^{n_{0}-1}\left(\frac{\pi w}{4 h}\right)^{\frac{n_{0}}{2}-1} \\
& +\frac{n_{0}-n_{1}}{\left(n_{1}-1\right)\left(n_{0}-1\right)},
\end{aligned}
$$

with $n_{0}$ and $n_{1}$ slopes in a $\log -\log$ plot of $F$ versus $\delta$ for power laws in two ranges $\delta<\delta^{*}$ and $\delta>\delta^{*}$, respectively (Khan and Sanmartin, 2014); model accuracy, when compared with numerical computations, proves quite reasonable, maximum deviations reaching upto $12 \%$ and $10 \%$ for ORDEM and MASTER respectively. The two straight lines in the log-log plot meet at the special point $\left(\delta^{*}, F^{*}\right)$. All four parameters in the model $\left(n_{0}, n_{1}, \delta^{*}, F^{*}\right)$ depend on orbit altitude $H$ and inclination. Fig. 1 shows an example of the dependence of these parameters on $H$ at $92^{\circ}$ and $63^{\circ}$ inclinations, for the MASTER model. In all cases debris diameter $\delta^{*}$ is close to $1 \mathrm{~mm}$. For ORDEM, debris flux might roughly be larger by one order of magnitude.

\section{The deorbiting dynamical equation}

In an orbiting frame there is a motional electric field $\mathbf{E}_{\mathrm{m}}=\left(\mathbf{v}-\mathbf{v}_{\mathrm{pl}}\right) \wedge \mathbf{B}$ in the highly conductive ambient plasma around, with plasma velocity $\mathbf{v}_{\mathrm{pl}}$ near-corrotational and negligible in LEO. A bare tape, its width not sensibly exceeding the electron Debye length, will collect electrons in the orbital motion limited regime (Sanmartin and Estes, 1999) over a segment coming out polarized positive. Ions are collected over the complementary segment, at too low rate because of the high $m_{i} / m_{e}$ mass ratio; effective current balance requires a plasma contactor, typically a Hollow Cathode (HC), to eject electrons at the cathodic end.

Both tether bias and current $I$ vary along the tether and are determined by solving a boundary value problem (Sanmartin et al., 1993). Current is negligible if $\mathbf{E}_{\mathbf{m}}$ points to the $\mathrm{HC}$, where electrons must be emitted; for a prograde (retrograde) orbit and $\mathbf{u}_{\mathbf{t}}$ the upwards vertical unit-vector, 

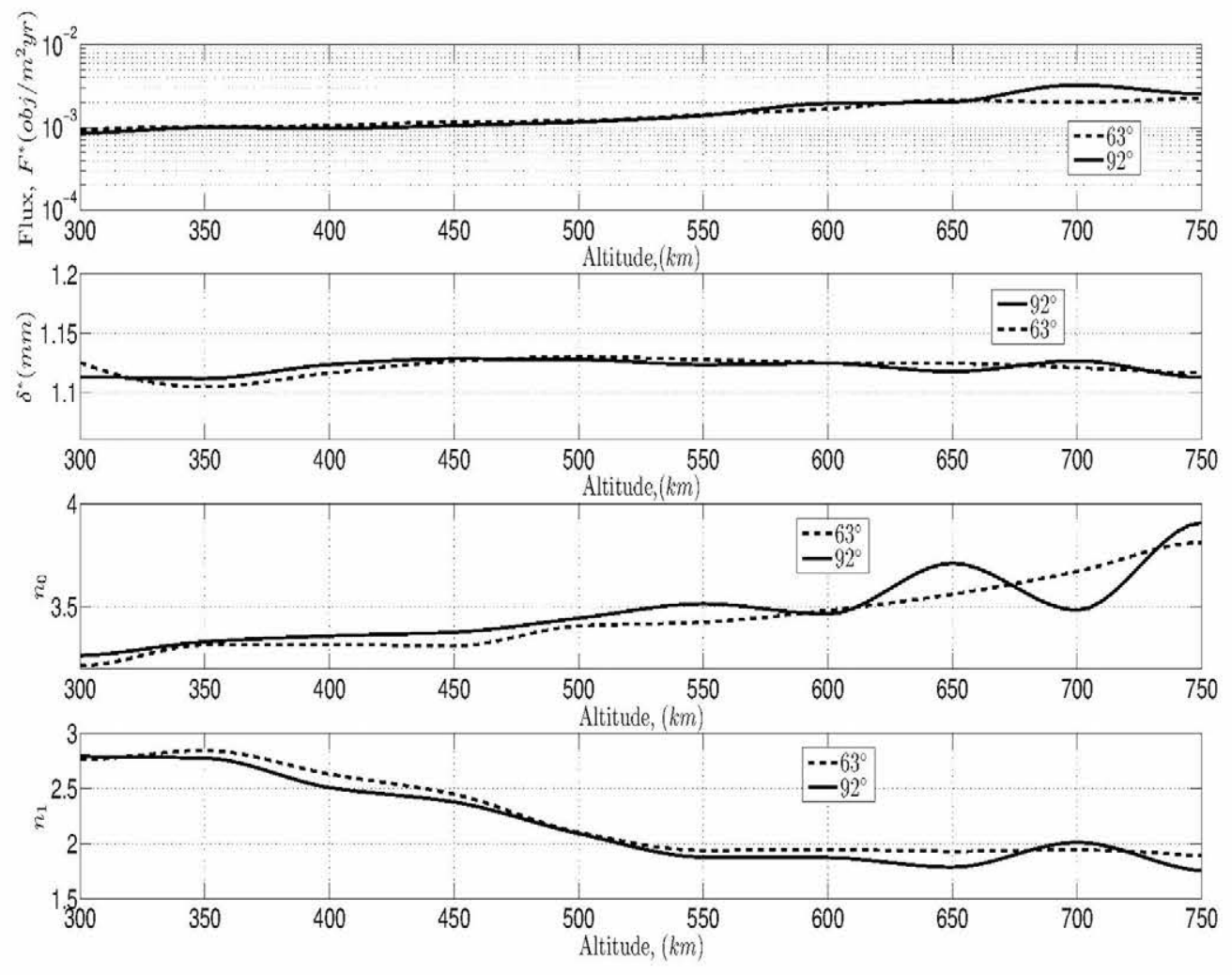

Fig. 1. Variation of $F^{*}, \delta^{*}, n_{0}$, and $n_{1}$ parameters in the MASTER flux model, for a range of $H$ values and given inclinations of $92^{\circ}$ (appropriate for satellite Cryosat) and $63^{\circ}$.
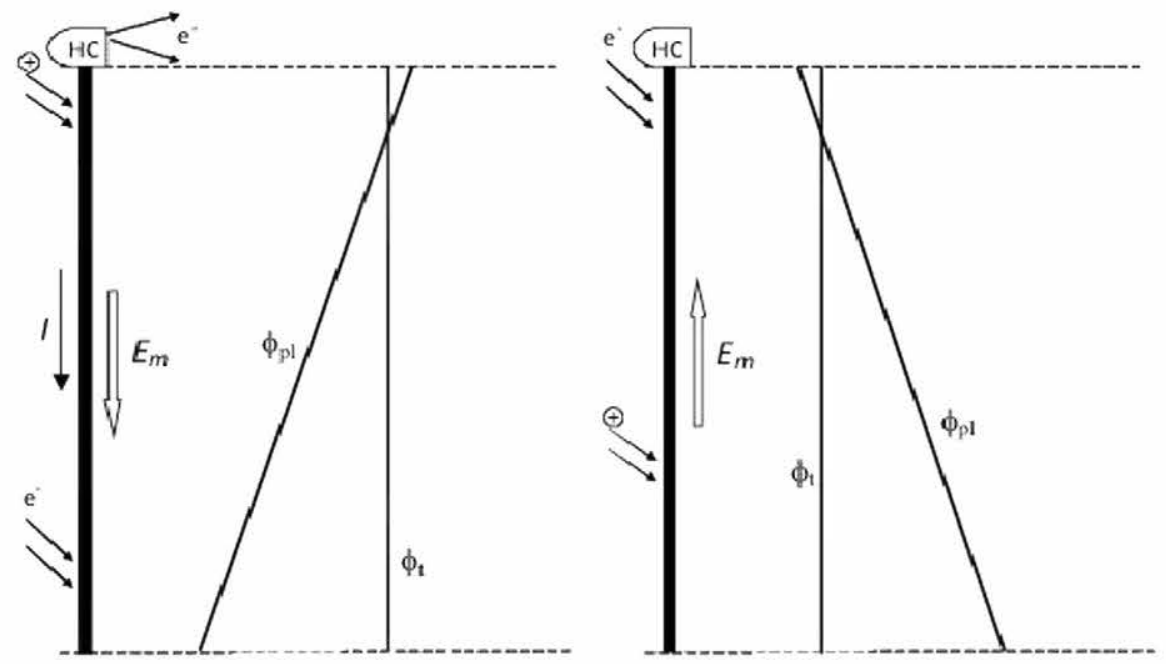

Fig. 2. Schematic of tether operation with the field $E_{m}$ reversing direction over a near polar, mostly retrograde orbit, with the HC at the top. For simplicity, ohmic effects are set negligible in the figure, leading to a potential profile $\phi_{t}$ constant along the tether; $\phi_{p l}$ is plasma potential.

the projection $E_{m}=\mathbf{u}_{\mathbf{t}} \cdot \mathbf{E}_{\mathbf{m}}$ is positive (negative) respectively. With the tether aligned with the local vertical, the $\mathrm{HC}$ must be placed at bottom (top) for prograde (retrograde) orbits. In the case of inclinations, say, from $80^{\circ}$ to $100^{\circ}, E_{m}$ changes sign as the Earth rotates under the orbital plane; only for the daily fraction where it has the right direction there is sensible current. Most of the time $E_{m}$ is positive (negative) for mostly prograde (retrograde) orbits; Fig. 2 shows a schematic of tether operation with the field $E_{m}$ reversing direction on a slightly retrograde (inclination above $90^{\circ}$ ) orbit.

Neglecting HC potential drop and ion collection, the length-averaged current along the tether $I_{a v}$, normalized by the short-circuit value $I_{s c}=\sigma_{c} h w E_{m}$, 
$\frac{I_{a v}}{I_{s c}} \equiv i_{a v}(\xi)$,

is a definite function of a ratio involving tether and ambient parameters (Sanmartin et al., 1993),

$$
\begin{aligned}
\xi & \equiv \frac{L}{h^{2 / 3} l^{1 / 3}}, \quad l \equiv \frac{9 \pi^{2}}{128} \frac{m_{e} \sigma_{c}^{2} E_{m}}{e^{3} n_{e}^{2}} \\
& \approx 2.38 \cdot 10^{18} \times \frac{E_{m} /(150 \mathrm{~V} / \mathrm{km})}{\left(n_{e} / 10^{5} \mathrm{~cm}^{-3}\right)^{2}}(\mathrm{~m}),
\end{aligned}
$$

where $\sigma_{c}$ is conductivity and $n_{e}$ electron plasma density and we are using aluminum tape. That function is exactly given as

$$
\begin{aligned}
& \left(1-i_{a v}\right) \xi=1, \quad \xi>4, \\
& \left(1-i_{a v}\right) \times \int_{0}^{1} \frac{d \varphi}{\sqrt{1-\left(1-\varphi^{3 / 2}\right)\left[\left(1-i_{a v}\right) \xi\right]^{3 / 2}}} \\
& =1, \quad \xi<4,
\end{aligned}
$$

both expressions meeting at $\xi=4, i_{a v}=3 / 4$ (Sanmartin et al., 2008). Approximations for (6b) are (6a) itself for $2<\xi<4$, say, and $i_{a v} \approx 0.3 \xi^{3 / 2}$ for $\xi<1$, this becoming exact for vanishing $\xi$. This last approximation is the no-ohmic limit,

$I_{a v} \approx \frac{2}{5} e n_{e} L \frac{2 w}{\pi} \sqrt{\frac{2 e E_{m} L}{m_{e}}}$.

For tape-tether design analysis, we consider a satellite of mass $M_{S}$ with a rigid tether along the vertical, in a circular orbit weakly perturbed by the Lorentz $\operatorname{drag} L I_{a v} \bar{u}_{t} \wedge \bar{B}$. The equation of motion is

$M_{S} \frac{d \mathbf{v}}{d t}+M_{S} \frac{\mu_{E}}{r^{2}} \frac{\mathbf{r}}{r}=L I_{a v} \mathbf{u}_{\mathbf{t}} \wedge \mathbf{B}$,

with $\mu_{E}$ the Earth's standard gravitational constant and $\mathbf{r}$ the position vector. The Lorentz drag makes the orbit slowly evolve through a long, spiraling sequence of quasi-circular orbits. Carrying out the scalar product with $\mathbf{v} \equiv d \mathbf{r} / \mathbf{d t}$ in Eq. (8) we have

$-M_{s} v d v / d t=-\sigma_{c} E_{m}^{2} w h L i_{a v}$,

where we used $\mathbf{E}_{\mathrm{m}} \approx \mathbf{v} \wedge \mathbf{B}$.

\section{Conductive tether design for a generic mission}

Eq. (9) can be rewritten as an equation of evolution for the orbit-altitude $H$ by using $v^{2} \approx v_{o r b}^{2}=\mu_{E} /\left(R_{E}+H\right)$ and introducing the mass $m_{c t}=\rho L w h$ of the conductive tether segment with density $\rho$, the full tether system possibly being twice as heavy,

$\frac{M_{S}}{m_{c t}} \frac{d H}{d t}=-2\left(R_{E}+H\right) \frac{\sigma_{c} E_{m}^{2}}{\rho v^{2}} \times i_{a v}(\xi)$,

which will hold over the fraction $f_{\tau}$ of orbital period having the motional field pointing away from the hollow cathode. We will take this into account by averaging (10) where negative over the orbits for a given day and introducing a factor $f_{\tau}$ on the right hand side. Outside the inclination range $80-100^{\circ}, f_{\tau}$ is close to unity.

We now derive a design formula for a generic mission combining the tether survival and deorbiting equation models (3) and (10). Dividing $d N_{c} / d t$ by $d H / d t$, there results an equation for the rate $d N_{c} d d H$,

$\frac{m_{c t}}{M_{S}} \frac{d N_{c}}{d H}=-\frac{L / 2}{R_{E}+H} \frac{\rho v^{2}}{\sigma_{c} E_{m}^{2}} \frac{\delta^{*} F^{*}}{f_{\tau} i_{a v}} \times G\left(n_{0}, n_{1}, \frac{\delta^{*}}{w}, \frac{w}{h}\right)$.

Writing $L \equiv \xi h^{2 / 3} l^{1 / 3}$ from Eq. (5) and integrating (11) from the initial altitude $H_{0}$ to some final altitude $H_{f}$ there results an equation for the product of $N_{f}$ (final value of $N_{c}$ ) and $m_{c t} / M_{S}$, which we wish to be small,

$\frac{m_{c t}}{M_{S}} \times N_{f}=\Pi\left(w, h, L / h^{2 / 3} ; H_{0}\right.$, inclination $)$,

$$
\begin{aligned}
\Pi \equiv & \int_{H_{f}}^{H_{0}} \frac{\left(R_{E}+H_{f}\right) d H / 2}{f_{\tau}\left(R_{E}+H\right)^{2}} \frac{\xi}{i_{a v}(\xi)} \frac{\rho v_{f}^{2}}{\sigma_{c} E_{m}^{2}} l^{1 / 3} h^{2 / 3} \delta^{*} F^{*} \\
& \times G\left(n_{0}, n_{1}, \frac{\delta^{*}}{w}, \frac{w}{h}\right)
\end{aligned}
$$

where $v_{f}=\sqrt{\mu_{E} /\left(R_{E}+H_{f}\right)}$ is the final velocity of the satellite, and parameters $F^{*}, \delta^{*}, n_{0}$ and $n_{1}$ are functions of altitude and inclination, as given in Fig. 1 for $92^{\circ}$ and $63^{\circ}$ inclinations.

We remark that $\Pi$, which depends separately on $L / h^{2 / 3}$ (through $i_{a v}$ ), w, and $h$, is time-independent and just involves tether geometry and orbit parameters. We consider de-orbiting down to $H_{f}=300 \mathrm{~km}$, where air-drag on the reasonably large tether front-area $\sim L w$, typically results in rapid reentry, while plasma density decreases below the ionospheric $F$ layer(s). An important feature of the design formula (13) is the ratio $\xi / i_{a v}$, which is just a function of the dimensionless variable $\xi$. Fig. 3 shows curves of $\xi, i_{a v}$ and $\xi / i_{a v}$ for a range of $\xi$ values; the indefinite increase of the ratio $\xi / i_{a v}(\xi)$ inside the integral for small and large $\xi$ will lead to some minimum of $\Pi$. As regards $w, \Pi$ varies as just $G$ in (13), which Eq. (3b) shows to decrease as $w$ increases; regarding $h$, however, $\Pi$ varies

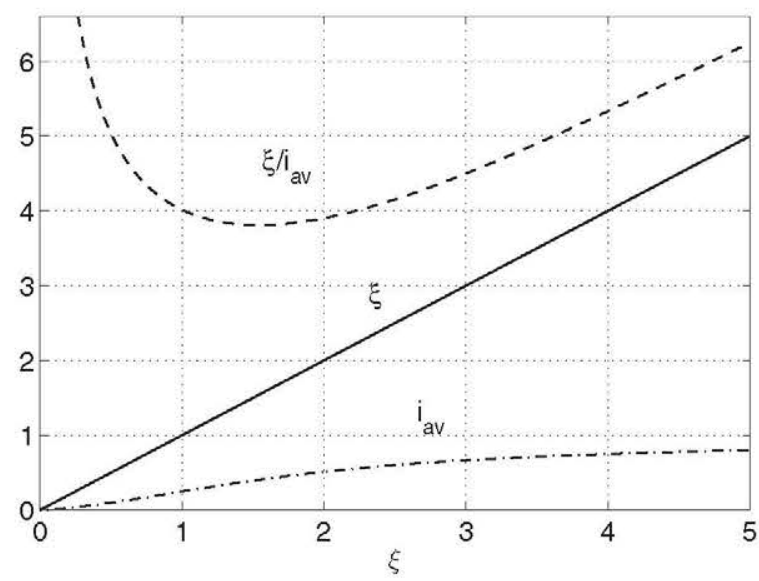

Fig. 3. Curves of $\xi, i_{a v}$ and $\xi / i_{a v}$ for a range of $\xi$ values. 
as $h^{2 / 3} \times G$, thus increasing with $h$ at $w$ large enough and decreasing at low enough $w$.

As regards de-orbit time $t_{f}$, carrying out the time integration in (10) and introducing

$\tau \equiv \int_{H_{f}}^{H_{0}}\left(\frac{E_{m}^{*}}{E_{m}(H)}\right)^{2} \frac{R_{E}+H_{f}}{\left(R_{E}+H\right)^{2}} \frac{d H / 2}{f_{\tau} i_{a v}}$,

$t_{\text {ref }}=\frac{\rho v_{f}^{2}}{\sigma_{c} E_{m}^{* 2}} \approx 5.3$ days $\approx 1.45 \times 10^{-2}$ years, $E_{m}^{*} \equiv 100 \mathrm{~V} / \mathrm{km}$,

these being convenient reference time and normalizing motional field respectively, yields

$$
\begin{aligned}
t_{f} & =\frac{M_{S}}{m_{c t}} \times t_{r e f} \times \tau\left(\frac{L}{h^{2 / 3}} ; H_{0}, \text { inclination }\right) \\
& \approx \frac{1.45 \times 10^{-2}}{m_{c t} / M_{S}} \times 1 \text { year } \times \tau,
\end{aligned}
$$

with $\tau$ depending on tape geometry through just the ratio $L / h^{2 / 3}$.

Carrying out the integrals defining $\Pi$ and $\tau$ in Eqs. (13) and (14) requires setting up the initial altitude $H_{0}$ and inclination, i.e. $720 \mathrm{~km}$ and either $92^{\circ}$ or $63^{\circ}$ as here considered. It next involves daily-averaged profiles in altitude $H$. II requires the 4 debris-flux model parameters given in Fig. 1 and the 2 ambient plasma profiles $E_{m}$ and $l$ (dependent on both $E_{m}$ and $n_{e}$ ) from the international IGRF and IRI models for $\mathbf{B}$ and $n_{e}$, both given in Fig. 4 for $92^{\circ}$ and $63^{\circ}$. Just the last two profiles are required for $\tau$.

An averaged solar flux of several 11-year cycles is considered for the ambient models. Notice that higher (lower) solar flux would make the electron density increase (decrease); the average current $I_{a v}$, which is dependent on $n_{e}$, would then increase and decrease for high and low solar flux, respectively. For the slightly retrograde Cryosat orbit at $92^{\circ}$ the daily-averaged field $E_{m}$ is negative about $56 \%$ of the time throughout, thus requiring a $\mathrm{HC}$ placed at the top and a nearly constant orbit factor $f_{\tau}=0.56$ in the integral for $\Pi$. For $63^{\circ}$ inclination, $f_{\tau}$ keeps close to 0.98 .

\section{Discussion of results}

Numerical results for $\tau$ versus $L / h^{2 / 3}$ for $92^{\circ}$ and $63^{\circ}$ inclinations are shown in Fig. 5. We introduced the convenient length $s$ given by

$s^{1 / 3} \equiv \xi l^{1 / 3} 1 / 3 \equiv L / h^{2 / 3}$,

with $\xi$ as in Eq. (5). Fig. 6 shows $\Pi$ versus $L / h^{2 / 3}$ for a range of $w$ and $h$ values and both inclinations; behavior is as discussed following Eq. (13). Note that $\tau$ decreases with increasing $s^{1 / 3}$ whereas $\Pi$ increases beyond its minimum. Results for $\tau$ and $\Pi$ at specific values convenient in the discussion that follows are later given in Tables 1 and 2 (for $h=40 \mu \mathrm{m}$ ).

Figs. 5 and 6 show clearly how moving from moderate to high inclination orbits makes tethers less efficient. Fig. 5 shows that tethers with given mass ratio will take longer in de-orbiting from higher inclination orbits, and Fig. 6 shows that tethers at higher inclinations will not reach as low values of product $\Pi$. Independently, the noticeable decrease of $\Pi$ with increasing $w$ suggest tethers will be more efficient in de-orbiting heavier satellites, requiring wider tapes, actual width being constrained, however, by electron collection lying in, or close to, the $O M L$ regime (Sanmartin and Estes, 1999), as mentioned in Section 3.

Optimum tape-design involves more than just one performance index. First, the conductive-tether mass ratio $m_{c t} / M_{S}$ must be small but not too small, because it naturally appears in the products $t_{f} \times m_{c t} / M_{S}$ and $N_{f} \times m_{c t} / M_{S}$, where $t_{f}$ and $N_{f}$ need be small too. With $\tau$ rapidly approaching its large-s limit independent of both $w$ and $h$ in Fig. 5, and $\Pi$ versus $s$ showing a minimum in Fig. 6, tether mass is written as $m_{c t}=\rho w h^{5 / 3} s^{1 / 3}$ for convenient scalable design; Fig. 6 suggests choosing $s^{1 / 3} \sim 2 \times 10^{6} \mathrm{~m}^{1 / 3}$ to allow minimum П. However, for $M_{S}$ scaling by two orders of magnitude from tens of $\mathrm{kg}$ reaching into tons, similar scaling in $m_{c t}$, with width $w$ keeping within or about OML range and thickness $h$ conveniently limited may be tough to attain. This suggests to use the Fig. 6 minimum being broad and flat to first increase $m_{c t}$ by moving $s^{1 / 3}$ by a factor of half an order
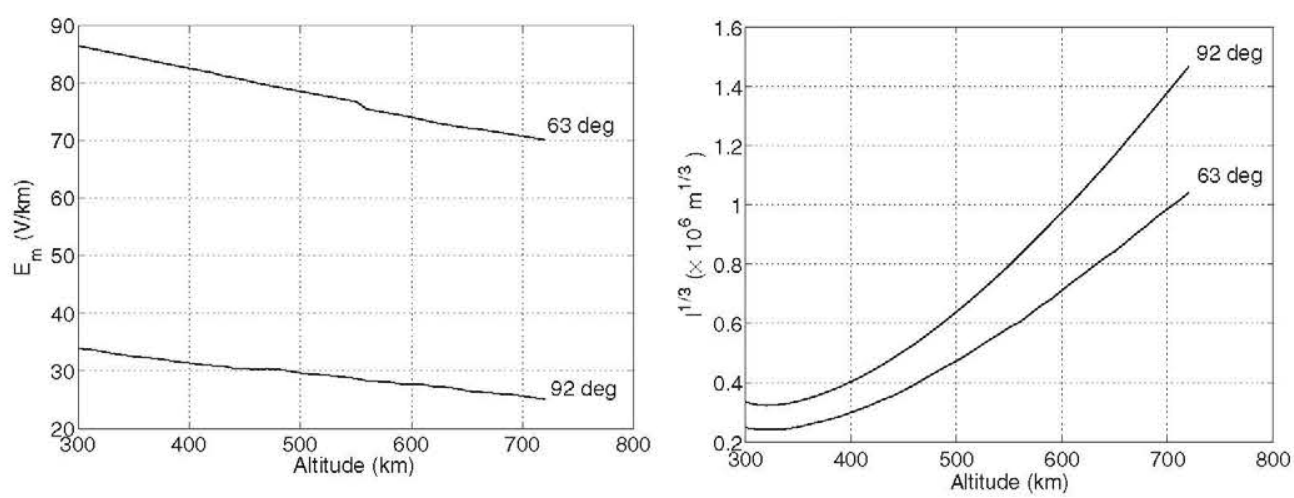

Fig. 4. Altitude profiles of $E_{m}$, and $l^{1 / 3}$ for both $63^{\circ}$ and $92^{\circ}$. 


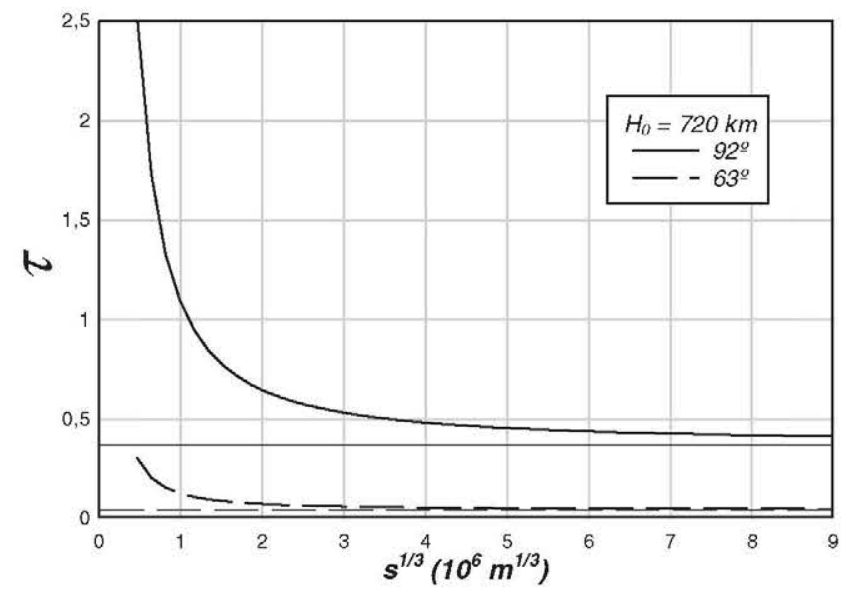

Fig. 5. Normalized de-orbit time $\tau$ in Eq. (16) from $720 \mathrm{~km}$, at $63^{\circ}$ and $92^{\circ}$.
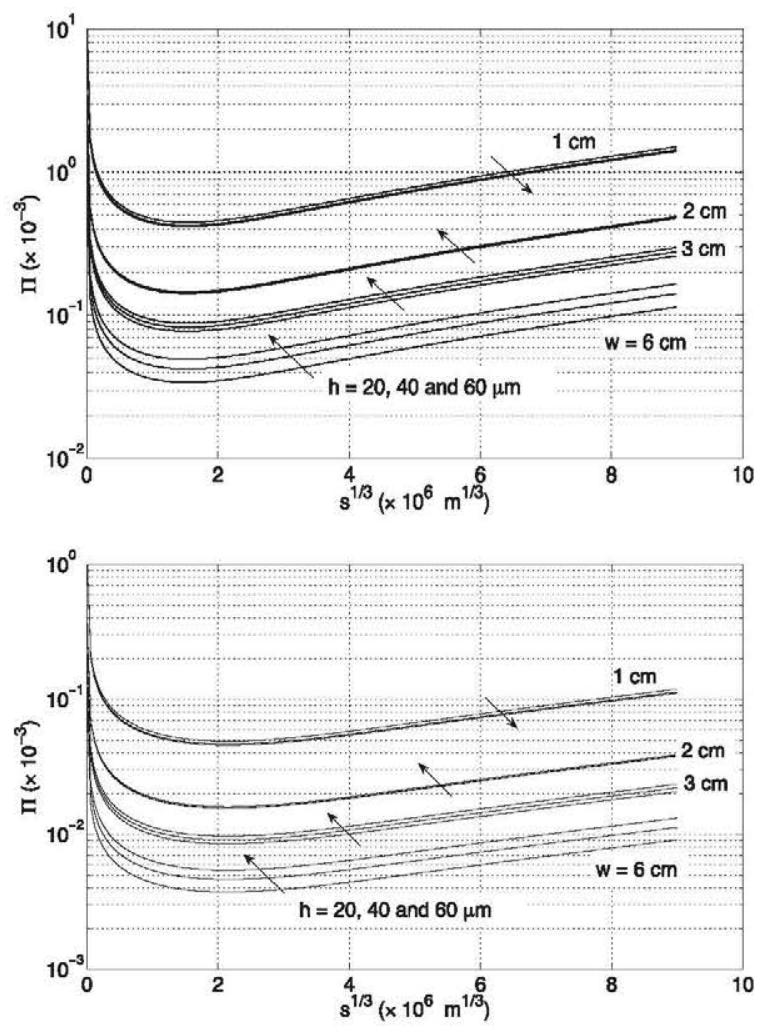

Fig. 6. Product $\Pi$ versus $L / h^{2 / 3}$ for a range of $w$ and $h$ values in deorbiting from $720 \mathrm{~km}$, at $63^{\circ}$ (bottom) and $92^{\circ}$ (top).

of magnitude to $s^{1 / 3}=6 \times 10^{6} \mathrm{~m}^{1 / 3}$ with little $\Pi$ increase. For the range $w=1-6 \mathrm{~cm}$, a corresponding $h$ range by factor $\left(10^{2} / 6\right)^{3 / 5} \approx 5.4$ suggests range values $h=10-54 \mu \mathrm{m}$, and yields $m_{c t}=0.75,75$ and $7.5 \mathrm{~kg}$ at upper and lower range ends, and mid-range $w=2.45 \mathrm{~cm}, h=23.2 \mu \mathrm{m}$, respectively. Choosing corresponding $M_{S}=37.5,375$, and $3750 \mathrm{~kg}$ values, the common $m_{c t} / M_{S}$ mass ratio is $2.0 \times 10^{-2}$, tether length $L$ being $2.8,4.9$, and $8.6 \mathrm{~km}$ respectively.

A second most important performance index is de-orbit time $t_{f}$, which characterizes the probability of a catastrophic collision of the satellite being de-orbited with other big S/C, its Area-time product, of interest in general considerations, being $t_{f} \times d^{2}$ for a representative S/C size $d \sim 2-3 \mathrm{~m}$, say. For the $s$ value above and $63^{\circ}$ inclination, Table 1 yields $\tau=5.06 \times 10^{-2}$, leading to just $t_{f}=13.4$ days in Eq. (16) for the mass-ratio $2.0 \times 10^{-2}$, independently of $M_{S}$.

A third index $t_{f} \times L$ characterizes a somehow minor accident, the collision of a big $\mathrm{S} / \mathrm{C}$ with the tether tape. The product $t_{f} L \times d$ represents an Area-time product characterizing the probability of such accident; for $d=2 \mathrm{~m}$ and again $63^{\circ}$ we find $203,357,621 \mathrm{~m}^{2}$ year for $M_{S}=37.5,375$ and $3750 \mathrm{~kg}$ respectively, well below values found in the literature (Nock et al., 2013). An obvious result, anyway, would be the tape being cut, aborting the de-orbit operation.

On the other hand, it is highly improbable that the collision would disrupt an operating S/C. The tape surface density may be relatively high for the extreme $54 \mu \mathrm{m}$ (aluminum) tape of the $3750 \mathrm{~kg}$ satellite, $\rho h=146 \mathrm{~g} / \mathrm{m}^{2}$, but the tape area impacting a $\mathrm{S} / \mathrm{C}$ would be small, $w \times d \sim 0.12 \mathrm{~m}^{2}$ for the corresponding $6 \mathrm{~cm}$ tape and $d \sim 2 \mathrm{~m}$ (as against $d^{2} \sim 4 \mathrm{~m}^{2}$ for the impact from a large sail or inflatable envelope). The resulting impact mass of $17.5 \mathrm{~g}$ does result, at a representative relative velocity of $10 \mathrm{~km} / \mathrm{s}$, i.e. $5 \times 10^{4} \mathrm{~J} / \mathrm{g}$, in impact energy under $10^{6} \mathrm{~J}$. If the $d=2 \mathrm{~m} \mathrm{~S} / \mathrm{C}$ weighs over 1 ton, the specific impact energy of less than $1 \mathrm{~J} / \mathrm{g}$ is well below the $15 \mathrm{~J} / \mathrm{g}$ threshold usually required for substantial damage (Nock et al., 2013).

A 4th and final performance index, specific for tethers, involves the probability of a tape cut by small debris. For the $w, h$ values leading to masses $0.75,7.5$, and $75 \mathrm{~kg}$, Fig. 6 and Table 2 (restricted to a representative $h=40 \mu \mathrm{m}$ value), roughly yield $\Pi \approx 0.8 \times 10^{-4}$, $0.2 \times 10^{-4}$, and $0.1 \times 10^{-4}$, leading to $N_{f} \approx 0.4 \times 10^{-2}$, $0.1 \times 10^{-2}$, and $0.05 \times 10^{-2}$, respectively. These very low probabilities resulting from use of ESA's MASTER flux, suggest use of NASA's, more conservative, ORDEM flux, about 10 times higher as mentioned in Section 2, would still yield low probabilities.

Consider now $92^{\circ}$ inclination, keeping $s^{1 / 3}=6 \times 10^{6} \mathrm{~m}^{1 / 3}$ and $m_{c t} / M_{S}=2 \times 10^{-2}$ as above. We would then have $\tau \approx 43.8 \times 10^{-2}$ in Table 1 , which is about 8.6 times greater

Table 1

Normalized de-orbit time $\tau$ as shown in Fig. $5 ; s^{1 / 3}$ is in units of $10^{6} \mathrm{~m}^{1 / 3}$.

\begin{tabular}{|c|c|c|c|c|c|c|c|c|c|c|}
\hline$i=92^{\circ}$ & & & & & & & $i=63^{\circ}$ & & & \\
\hline$s^{1 / 3}$ & 6 & 9 & 12 & 15 & 18 & $\infty$ & 6 & 9 & 12 & $\infty$ \\
\hline$\tau$ & 0.44 & 0.41 & 0.40 & 0.395 & 0.39 & 0.37 & 0.0505 & 0.048 & 0.0468 & 0.0437 \\
\hline
\end{tabular}


Table 2

Product $\Pi$ as shown in Fig. 6 , for $h=40 \mu \mathrm{m} ; w$ is in $\mathrm{cm}$ and $s^{1 / 3}$ in units of $10^{6} \mathrm{~m}^{1 / 3}$

\begin{tabular}{|c|c|c|c|c|c|c|c|c|}
\hline \multirow[t]{4}{*}{$w$} & \multicolumn{8}{|c|}{$\Pi\left(10^{-4}\right)$} \\
\hline & \multicolumn{4}{|c|}{$i=92^{\circ}$} & \multicolumn{4}{|c|}{$i=63^{\circ}$} \\
\hline & \multicolumn{8}{|l|}{$s^{1 / 3}$} \\
\hline & 3 & 6 & 9 & 12 & 3 & 6 & 9 & 12 \\
\hline 1 & 5.09 & 8.68 & 13.38 & 19.79 & 0.49 & 0.72 & 1.06 & 1.50 \\
\hline 2 & 1.71 & 2.91 & 4.49 & 6.64 & 0.16 & 0.24 & 0.36 & 0.50 \\
\hline 3 & 0.99 & 1.69 & 2.60 & 3.84 & 0.095 & 0.14 & 0.21 & 0.29 \\
\hline 6 & 0.51 & 0.86 & 1.33 & 1.96 & 0.048 & 0.072 & 0.11 & 0.15 \\
\hline
\end{tabular}

than the corresponding $63^{\circ}$ value, leading to the same increase in time $t_{f}$. Also, increases in $\Pi$ from Table 2 reach over one order of magnitude. A reasonable design choice would make mass-ratio and de-orbit time share the loss in performance by allowing $m_{c t} / M_{S}$ to increase by a factor, say, 2.1 and $t_{f}$ by a factor 4.1 , yielding $m_{c t} / M_{S} \approx 4.2 \times 10^{-2}$ and $t_{f} \approx 54.9$ days, which still is two orders of magnitude shorter than the 25 year bound on de-orbit time first established by NASA. For the $M_{S}=37.5 \mathrm{~kg}$ case above, we may get twice tether mass, $1.5 \mathrm{~kg}$, by just moving $w$ from 1 to $2 \mathrm{~cm}$, and keeping $h$ and thus $L$; this results in an increase of $t_{f} L$ by the 4.1 factor above. As regards the $N_{f}$ probability, the increase in the value of $\Pi$ resulting from moving to $92^{\circ}$ inclination is partly compensated by the larger mass ratio and the move to the wider tape, which reduces $\Pi$ itself; we find $N_{f} \approx 0.7 \times 10^{-2}$, as compared with the value $0.4 \times 10^{-2}$ for $63^{\circ}$. Proceeding similarly for the $M_{S}=375 \mathrm{~kg}$ case, we double tether mass to $15 \mathrm{~kg}$ by moving to $w=4.9 \mathrm{~cm}$ and again keeping $h$ and $L$, and increasing $t_{f} L$ by the 4.1 factor. We then find $N_{f} \approx 0.25 \times 10^{-2}$, as compared with the result $0.1 \times 10^{-2}$ for $63^{\circ}$.

The case for $M_{S}=3750 \mathrm{~kg}$, and in general the multi-ton tether case is different because any sensible $w$ increase over $6 \mathrm{~cm}$, say, would make collected current drop below OML values as used from start (Estes and Sanmartin, 2000). Doubling tether mass at $w$ constant requires doubling $h^{5 / 3}$, i.e. increasing $h$ by about 1.5 to $81 \mu \mathrm{m}$, and thus $L$ by a 1.32 factor, and $t_{f} L$ increased accordingly. We again find $N_{f}$ about $0.25 \times 10^{-2}$, as compared with the result $0.05 \times 10^{-2}$ for $63^{\circ}$.

\section{Conclusions}

Universal tape-tether design, involving selection of conductive-segment length $L$, width $w$, and thickness $h$, is here discussed for particular missions: initial orbit of $720 \mathrm{~km}$ altitude and $63^{\circ}$ and $92^{\circ}$ inclinations, and 3 disparate $M_{S}$ values, $37.5,375$, and $3750 \mathrm{~kg}$, design proving scalable. Performance is gauged by requiring very low both probability $N_{f}$ of tether cut by small debris and ratio of conductive-segment mass $m_{c t}$ to $M_{S}$, and short de-orbit time $t_{f}$ and low $t_{f} L$. At mid-inclination and $2 \% m_{c t} / M_{S}$ mass-ratio, de-orbit time takes about 2 weeks and $N_{f}$ is a small fraction of $1 \%$, with tape dimensions ranging from
2.8 to $8.6 \mathrm{~km}, 1$ to $6 \mathrm{~cm}$, and 10 to $54 \mu \mathrm{m}$. Performance drop from middle to high inclination proved moderate: if allowing for twice as large $m_{c t} / M_{S}$, increases are reduced to a factor of 4 in $t_{f}$ and a slight one in $N_{f}$; multi-ton $\mathrm{S} / \mathrm{C}$ are more requiring, however, because efficient orbital-motion-limited collection restricts tape-width values, resulting in tape length (slightly) increasing too.

Although electrical power is involved, the tether system, in addition to allow low-mass scalable design, is a passive system, needing neither propellant nor auxiliary power and arising from just thermodynamics and thus being reliable as air drag; low Work Function materials being explored might actually do away with use of the Hollow Cathode plasma contactor at the cathodic end. The bare-tether tape geometry would also do away with the need of a multi-line tether to resist the small debris menace (Forward and Hoyt, 1995). It de-orbits fast, about two weeks for initial altitudes $700-750 \mathrm{~km}$ at mid-inclinations and two months at high inclination; estimates show de-orbit time keeping well as fraction of year for altitudes around $1000 \mathrm{~km}$, high inclination conditions. The key pending issue is a thorough in-orbit demonstration of tether deployment and $\mathrm{HC}$ operation.

\section{Acknowledgments}

This work was supported by the European Commission FP7/Space Project 262972 (BETs), the Ministry of Science and Innovation of Spain (BES-2009-013319 FPI Grant), and Universidad Politécnica de Madrid (Research Grant RR01 2009).

\section{References}

Ahedo, E., Sanmartin, J.R., 2002. Analysis of bare-tether systems for deorbiting LEO satellites. J. Spacecraft Rockets 39 (2), 198-205.

Estes, R.D., Sanmartin, J.R., 2000. Cylindrical Langmuir probes beyond the orbital-motion-limited regime. Phys. Plasmas 7, 4320 4325.

Flegel, S., et al., 2009. The MASTER-2009 Space Debris Environment Model. Darmstdat, ESA SP-672.

Forward, R.L., Hoyt, R.P., 1995. Failsafe multiline hoytether lifetimes. San Diego, CA, AIAA, ASME, SAE, and ASEE, Joint Propulsion Conference and Exhibit.

Forward, R.L., Hoyt, R.P., Uphoff, C., 1998. The terminator tether: a near-term commercial application of the NASA/MSFC ProSEDS experiment. In: Proceeding of the Tether Technology Interchanging Meeting, NASA CP-1998-(206900), p. 109. 
Gilchrist, B. et al., 2002. The use of electrodynamic tethers for orbit maintenance and deorbit of large spacecraft: a trade study of the NASA GLAST mission. AIAA Paper, 2002-4044.

Kessler, D.J., Cour-Palais, B.G., 1978. Collision frequency of artificial satellites: the creation of a debris belt. J. Geophys. Res. 83 (A6), 2637 2646.

Khan, S.B., Sanmartin, J.R., 2013. Survival probability of round and tape tethers against debris impact. J. Spacecraft Rockets 50 (3), 603-608.

Khan, S.B., Sanmartin, J.R., 2014. Analysis of tape tether survival in LEO against orbital debris. Adv. Space Res, 53 (9), 1370-1376.

Liou, J.-C., et al., 2002. The new NASA orbital debris engineering model ORDEM2000. NASA STI/Recon Technical Report N 2, 51086.

Nock, K.T., Aaron, K.M., McKnight, D., 2013. Removing orbital debris with less risk. J. Spacecraft Rockets 50 (2), 365-379.
Pardini, C., Hanada, T., Krisko, P., 2006. Potential benefits and risks of using electro-dynamic tethers for end-of-life de-orbit of LEO spacecraft, IADC: 06-02.

Pardini, C., Hanada, T., Krisko, P., 2009. Benefits and risks of using electro-dynamic tethers to de-orbit spacecraft. Acta Astronaut. 64, $571-588$.

Sanmartin, J.R., Estes, R.D., 1999. The orbital-motion-limited regime of cylindrical Langmuir probes. Phys. Plasmas 6, 395-405.

Sanmartin, J.R., Martinez-Sanchez, M., Ahedo, E., 1993. Bare wire anodes for electrodynamic tethers. J. Propul. Power 9 (3), 353- 360.

Sanmartin, J.R. et al., 2008. Electrodynamic tether at Jupiter-I: capture operation and constraints. IEEE Trans. Plasma Sci. 36 (5), 2450-2458.

Van der Heide, E.J., Kruijff, M., 2001. Tethers and debris mitigation. Acta Astronaut. 48, 503-516. 